\title{
A COHESIVE APPROACH TO THIN-SHELL FRACTURE AND FRAGMENTATION
}

\author{
$\underline{\text { Fehmi Cirak }}^{*}$, Michael Ortiz**, Anna Pandolfi*** \\ ${ }^{*}$ CACR, Caltech, Pasadena, CA 91125, U.S.A. \\ ${ }^{* *}$ GALCIT, Caltech, Pasadena, CA 91125, U.S.A. \\ ${ }^{* *}$ DIS, Politecnico di Milano, 20133 Milano, Italy
}

\begin{abstract}
Summary We develop a finite-element method for the simulation of dynamic shell fracture and fragmentation based on cohesive models of fracture. We assume the shells to be thin and to obey the Kirchhoff-Love theory. The shell is spatially discretized by means of subdivision shell elements. Fracture is allowed only along element edges and is assumed to be governed by a cohesive law. When coupled to the shell kinematics, the cohesive model accounts both for in-plane or tearing, shearing, and bending or hinge modes of fracture. In order to follow the propagation and branching of cracks, subdivision shell elements are pre-fractured $a b$ initio. Prior to crack nucleation, crack opening is constrained by means of a penalty method in implicit calculations, or by a projection or displacement averaging method in explicit calculations.
\end{abstract}

\section{OVERVIEW}

The experimental and analytical investigation of fracture and fragmentation of thin-plates and shells has elicited considerable interest in the past. The analytic models proposed to date often introduce ad-hoc approximations regarding the likely failure modes and are restricted to a few regular geometries, loading conditions, and constitutive models. By contrast, numerical simulation of fracture and fragmentations of shells and plates has received comparatively less attention. A notable exception is furnished by the finite element analysis of "surface-cracked" plates and shells [2]. In [1], Rice and Levy introduced the concept of a line-spring with stretching and bending resistance for the analytical treatment of plates with a surface crack, and computed its compliance from fracture mechanics and energetic considerations. In the finite element context the line-spring is inserted at the location of the surface-crack in the plate or shell in order to compute quantities relevant for fracture mechanics [2].

In the present work, fracture initiation and propagation is considered as a progressive failure phenomenon in which the separation of the crack flanks is resisted by cohesive tractions. The relationship between the crack opening displacements and the tractions is given by a cohesive model. An appealing feature of the cohesive model is that it provides a complete theory of fracture without presuming a particular bulk material behavior or geometry of the specimen and the extend of the crack growth.

For three-dimensional solids, cohesive laws may conveniently be encoded into cohesive elements in the form of double surfaces (e. g., $[5,7,6,8]$ and references therein). The opening of the cohesive elements is compatible with the deformation of the adjacent volume elements and is subject to a unilateral closure constraint. Cohesive elements may be inserted adaptively upon the attainment of a critical stress condition on the interelement boundary $[7,8]$. The insertion of cohesive elements introduces new surfaces into the mesh, which undergoes complex topological transitions as a result. For threedimensional solids these transitions can be classified exhaustively [8], and appropriate actions may be taken in order to update the representation of the mesh in each case.

Certain aspects of the mechanics of shells greatly compound the integration of cohesive theories of fracture. Thus, within the framework of Kirchhoff-Love theory the strain energy density of thin-shells is expressed in terms of the first and second fundamental forms of the shell middle surface. Therefore, a conforming finite element discretization requires smooth shape functions belonging to the Sobolev space $H^{2}$. Cirak, Ortiz, and Schröder [3,4] proposed a new type of discretization based on the concept of subdivision surfaces which delivers smooth $H^{2}$ shape functions on unstructured meshes in a particularly natural and efficient way. In addition to several other advantages, an appealing feature of the subdivision elements is that the sole unknowns in the finite element solution are the nodal displacements. However, this comes at a certain cost, namely, that the subdivision shape functions are non-local in the sense that the displacement field within one element depends on the displacements of the nodes attached to the element and the immediately adjacent ring of nodes in the mesh.

The natural extension of the cohesive element concept to shells consists of inserting cohesive elements at interelement edges, and constraining the opening of the cohesive elements to conform to the deformation of the middle surface of the shell and its normal. This approach allows for fracture both in an in-plane or tearing mode, a shearing mode, or a bending of hinge mode. However, within a subdivision-element framework the essential difficulty resides in making the scheme adaptive, in the sense of inserting cohesive elements in an otherwise conforming mesh upon the attainment of some appropriate critical condition. Thus, the non-locality of the displacement interpolation renders the tracking of the topological transitions induced by the insertion of cohesive elements unmanageably complex. In order to sidestep this difficulty we simply fragment ab initio all the element edges by duplication of common nodes. In implicit calculations, element conformity prior to fracture is enforced by the addition of a penalty term to the energy. Alternatively, in explicit dynamics calculations conformity is readily enforced by a displacement averaging technique. 

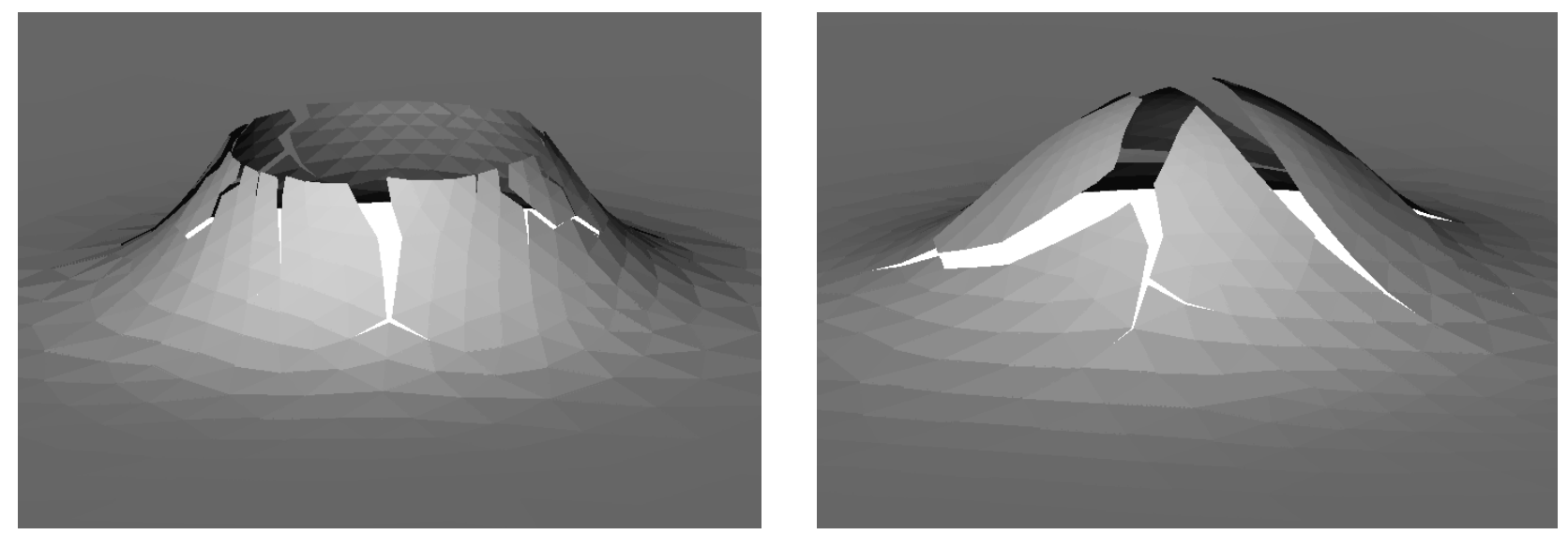

Figure 1. (a) Detail of the petalling formation in a perforated plate. (b) Star-shaped crack formation in a solid plate.

\begin{abstract}
APPLICATIONS
The present framework can be applied to a variety of thin plate and shell fracture and fragmentation problems, such as the perforation of thin-plates impacted by fast moving projectiles. As an example of application, we simulate the experiments of Landkof and Goldsmith [9] concerned with the petalling failure of circular aluminum (Al 2024-0) plates with and without initial holes struck by hard-steel conical projectiles.

In computations we consider a solid and a perforated plate with a $6.35 \mathrm{~mm}$ diameter hole. The plate thickness is $3.175 \mathrm{~mm}$ in both cases. The striking velocity is $v=320 \mathrm{~m} / \mathrm{s}$. The bulk material response of the aluminum plates is assumed to obey a rate dependent $\mathrm{J} 2$-plasticity model. To simulate the effect of the projectile impact, we apply a pressure history over the impact area corresponding to the force exerted by the conical tip of the projectile. The impact force has been computed from the deceleration $\Delta v$ of the projectile, as measured in the experiments.

The results of the dynamical analysis of the solid and perforated plate are collected in Fig. 1. For the perforated plate, in keeping with theory and experiment [9], the early states of penetration are characterized by an enlargement of the hole, followed by crack propagation, and final bending of trapezoidal petals. A detail of the central part of the perforated plate at the end of the calculation is shown in Figure 1a. Of course, the fine details of the fracture patterns are to some extend stochastic and are likely to exhibit considerable scatter. For present purposes it suffices to point out that the numerical method allows for complex fracture patterns including branching and fragmentation. As observed in [9], for solid plates petalling starts with the formation of a star-shaped crack at the impact point, and the subsequent propagation of radial cracks. The resulting triangular petals rotate about a continuously shifting circular plastic hinge in order to allow the passage of the projectile. A detail of the central part of the plate after the formation of the star shaped crack is shown in Fig. 1b. The branching events in some of the radial cracks are particularly noteworthy.
\end{abstract}

\title{
References
}

[1] J.R. Rice and Levy N. The part-through surface crack in an elastic plate. Journal of Applied Mechanics, 39(1):185-194, 1972.

[2] D.M. Parks and White C.S. Elastic-plastic line-spring finite elements for surface-cracked plates and shells. Journal of Pressure Vessel Technology, 104(4):287-292, 1982.

[3] F. Cirak, M. Ortiz, and P. Schröder. Subdivision surfaces: A new paradigm for thin-shell finite-element analysis. International Journal for Numerical Methods in Engineering, 47(12):2039-2072, 2000.

[4] F. Cirak and M. Ortiz. Fully $C^{1}$-conforming subdivision elements for finite deformation thin-shell analysis. International Journal for Numerical Methods in Engineering, 51:813-833, 2001.

[5] X.-P. Xu and A. Needleman. Numerical simulations of fast crack growth in brittle solids. Journal of the Mechanics and Physics of Solids, 42(9):1397-1434, 1994.

[6] M. Ortiz and A. Pandolfi. A class of cohesive elements for the simulation of three-dimensional crack propagation. International Journal for Numerical Methods in Engineering, 44:1267-1282, 1999.

[7] G. T. Camacho and M. Ortiz. Computational modelling of impact damage in brittle materials. International Journal of Solids and Structures, 33(20-22):2899-2938, 1996.

[8] A. Pandolfi and M. Ortiz. An efficient adaptive procedure for three-dimensional fragmentation simulations. Engineering with Computers, 18(2):148-159, 2002.

[9] B. Landkof and W. Goldsmith. Petalling of thin, metallic plates during penetration by cylindro-conical projectiles. International Journal of Solids and Structures, 21(3):245-266, 1985. 